\title{
$p$-ADIC DIFFERENCE-DIFFERENCE LOTKA-VOLTERRA EQUATION AND ULTRA-DISCRETE LIMIT
}

\author{
SHIGEKI MATSUTANI
}

(Received 28 August 2000 and in revised form 8 March 2001)

\begin{abstract}
We study the difference-difference Lotka-Volterra equations in $p$-adic number space and its $p$-adic valuation version. We point out that the structure of the space given by taking the ultra-discrete limit is the same as that of the $p$-adic valuation space. Since ultra-discrete limit can be regarded as a classical limit of a quantum object, it implies that a correspondence between classical and quantum objects might be associated with valuation theory.
\end{abstract}

2000 Mathematics Subject Classification. 35Q53, 12J20, 12H25, 81Sxx.

1. Introduction. In soliton theory, difference-difference equations, whose domain space-time are given by integers, and the ultra-discrete difference-difference equations, whose, all, domain and range are given by integers, are currently studied $[12,24,25]$. In the field, it remains the problem of what is the ultra-discrete.

On the other hand, recently number theory and physics might be considered as a missing link of each other. For example, a set of geodesics in a compact Riemannian surface with genus $g \geq 2$ are investigated in the framework of chaos because any geodesics, or orbits, part from each other due to its negative curvature [1, 22] (whereas the Jacobi varieties of the Riemannian surfaces are completely classified by a soliton equation [18]). By quantization of the orbits, there appears quantum chaos and, as it is very mysterious, its partition function has a very similar structure as zeta functions in number theory $[1,22]$. (Level statistics in quantum chaos is also connected with integrable systems [21].) Using the resemblance of zeta functions, Connes proposed a kind of unification of number theory and quantum statistical physics in order to solve the Riemannian conjecture of the zeta-function [2, 5].

Further on the discrimination problem of integrability of Hamiltonian systems, there appears Galois theory in the category of differential equations [17], which plays the same role in the category of the number theory.

Thus in order to know what is the integrability or quantization, it is not surprising that there appears integer theory in physics. In fact, there are many other studies pointing out that the $p$-adic number theory and non-archimedean valuation theory are closely related to statistical and quantum physics [3, 20, 26], even though $p$-adic space has a metric which differs from euclidean sense. These correspondences might imply that there is a deep hidden symmetry behind physics and number theory and give a novel step to mathematical physics.

Thus I believe that it is very important to interpret such current development of soliton theory using $p$-adic number theory and non-archimedean valuation theory. In 
this article, we mainly deal with the Lotka-Volterra equation as a typical differencedifference soliton equation. We show that even in $p$-adic space of the number theory, the difference-difference Lotka-Volterra equation has mathematical meanings and has nontrivial solutions in Proposition 5.2. It means that in $p$-adic space, we can deal with the soliton equation as we do in real number space. In Proposition 5.3, we consider the $p$-adic valuation version of the $p$-adic equation. It is surprising that the formal structure of the equation is the same as the ultra-discrete difference-difference LotkaVolterra equation. We will compare the ultra-discrete soliton system and the $p$-adic valuation version of the $p$-adic soliton system. It is shown that the ultra-discrete limit is similar to the $p$-adic valuation and should be regarded as a non-archimedean valuation.

Since the ultra-discrete limit can be considered as a classical limit of a quantized object or zero temperature limit of statistical mechanical object, the relation between ultra-discrete limit and $p$-adic valuation implies that correspondence between classical and quantum objects might be concerned with valuation theory.

In this article we start with a preliminary of $p$-adic number theory in Section 2. Sections 3 and 4 review the recent development of difference-difference soliton theory and the ultra-discrete soliton theory for the Lotka-Volterra equations, respectively, [25]. In order to compare the $p$-adic valuation with the ultra-discrete limit, we will slightly modify the definitions of the ultra-discrete limit given in [23, 25]. In Section 5, after we formally construct a $p$-adic difference-difference Lotka-Volterra equation, we prove its existence and explicit forms of its solutions. We show the resemblance between the $p$-adic valuation of the $p$-adic difference-difference Lotka-Volterra equation and the ultra-discrete difference-difference Lotka-Volterra equation. In order to study the ultra-discrete system from the point of view of valuation theory, we define the ultrametric induced from the ultra-discrete limit. Section 6 is devoted to investigate the space of ultra-discrete limit. We comment upon physical and mathematical meanings of the ultra-discrete limit and ultrametric.

2. Preliminary: $p$-adic space. We review the $p$-adic valuation and its related topics for a prime number $p[3,4,14,16,20,26]$. For a rational number $u \in \mathbb{Q}$ which is given by $u=(v / w) p^{m}$ ( $v$ and $w$ are coprime to the prime number $p$ and $m$ is an integer), we define a symbol $[[u]]_{p}:=p^{m}$. Here we define the $p$-adic valuation.

DEFINITION 2.1. We define a map from $\mathbb{Q}$ to a set of integers $\mathbb{Z}$; for $u \in \mathbb{Q}$,

$$
\operatorname{ord}_{p}(u):=\log _{p}[[u]]_{p}, \quad \text { for } u \neq 0, \quad \operatorname{ord}_{p}(u):=\infty, \quad \text { for } u=0 .
$$

We call its image $p$-adic valuation of $u$.

This valuation has the following properties $\left(\mathrm{I}_{p}\right)$.

Proposition $2.2\left(\mathrm{I}_{p}\right)$. For $u, v \in \mathbb{Q}$,

(1) $\operatorname{ord}_{p}(u v)=\operatorname{ord}_{p}(u)+\operatorname{ord}_{p}(v)$.

(2) $\operatorname{ord}_{p}(u+v) \geq \min \left(\operatorname{ord}_{p}(u), \operatorname{ord}_{p}(v)\right)$.

If $\operatorname{ord}_{p}(u) \neq \operatorname{ord}_{p}(v), \operatorname{ord}_{p}(u+v)=\min \left(\operatorname{ord}_{p}(u), \operatorname{ord}_{p}(v)\right)$.

Proof. From the definition, they are obvious [3, 4, 14, 20, 26]. 
The property $\left(\mathrm{I}_{p}\right)(1)$ means that $\operatorname{ord}_{p}$ is a homomorphism from the multiplicative group $\mathbb{Q}^{\times}$of $\mathbb{Q}$ to the additive group $\mathbb{Z}$. The $p$-adic metric is defined by $|v|_{p}:=p^{-\operatorname{ord}_{p}(v)}$ which has the following properties ( $\left.\mathrm{II}_{p}\right)$ (see $\left.[4,26]\right)$.

Proposition $2.3\left(\mathrm{II}_{p}\right)$. For $u, v \in \mathbb{Q}$,

(1) if $|v|_{p}=0$ then $v=0$.

(2) $|v|_{p} \geq 0$.

(3) $|v u|_{p}=|v|_{p}|u|_{p}$.

(4) $|u+v|_{p} \leq \max \left(|u|_{p},|v|_{p}\right) \leq|u|_{p}+|v|_{p}$.

Proof. From the definition, they are also obvious [3, 4, 14, 20, 26].

REMARK 2.4. (1) The $p$-adic field $\mathbb{Q}_{p}$ is given as a completion of $\mathbb{Q}$ with respect to this metric so that properties $\left(\mathrm{I}_{p}\right)$ and $\left(\mathrm{II}_{p}\right)$ survive for $\mathbb{Q}_{p}$.

(2) The integer part $\mathbb{Z}_{p}$ of $\mathbb{Q}_{p}$ is a "localized ring" and has only prime ideals $\{0\}$ and $p \mathbb{Z}_{p}$.

(3) As the properties of $p$-adic metric, the convergence condition of the series $\sum_{m} x_{m}$ is identified with the vanishing condition of the sequence $\left|x_{m}\right|_{p} \rightarrow 0$ for $m \rightarrow \infty$ due to the relationship [4, 16, 26],

$$
\left|\sum_{m: \text { finite sum }} x_{m}\right|_{p}=\max \left|x_{m}\right|_{p} .
$$

REMARK 2.5. We define $|u|_{\infty}$ as a natural metric or absolute value over the real field $\mathbb{R},|u|_{\infty}:=|u|$, and $\mathbb{R}$ is regarded as a field over the $\infty$ point of prime numbers; we will denote $\mathbb{R}$ by $\mathbb{Q}_{\infty}$. Then we have a relation for any nonzero $u \in \mathbb{Q}$,

$$
\prod_{p \in \mathfrak{A}}|u|_{p}=1
$$

where $\mathcal{A}$ is $\{2,3,5,7,11,13, \ldots, \infty\}$. This is an adelic property of $p$-adic metric $[16,26]$.

3. Difference-difference Lotka-Volterra equation. In this section, we deal with the difference-difference Lotka-Volterra equation [12, 25]. Along the line of the arguments of [25], it can be regarded as the difference-difference analogue of the Korteweg-de Vries (KdV) equation,

$$
\partial_{t} u+6 u \partial_{s} u+\partial_{s}^{3} u=0
$$

where $\partial_{t}:=\partial / \partial t$ and $\partial_{s}:=\partial / \partial s$ and $u=u(s, t)$ whose domain $(t, s)$ is $\mathbb{R}^{2}$.

DEFINITION 3.1. The difference-difference Lotka-Volterra equation is given as [12],

$$
\frac{c_{n}^{m+1}}{c_{n}^{m}}=\frac{1+\delta c_{n-1}^{m}}{1+\delta c_{n+1}^{m+1}},
$$

for a real parameter $\delta \in \mathbb{R}$ and $\left\{c_{n}^{m} \in \mathbb{R} \mid n, m \in \Omega\right\}$, where $\Omega$ is a subset of $\mathbb{Z}^{2}$.

We note that this equation (3.2) has the trivial solutions in which $c_{n}^{m}=c$ for a constant $c$ and all $n$ and $m$ in $\Omega$. 
Equation (3.2) is related to the bilinear difference-difference equation $(3.3)[8,11$, $12,25]$.

LEMMA 3.2. For the set $\left\{\tau_{n}^{m}\right\}$ whose elements hold the bilinear relation,

$$
\tau_{n+1}^{m+1} \tau_{n}^{m}-(1+\delta) \tau_{n+1}^{m} \tau_{n}^{m+1}+\delta \tau_{n-1}^{m} \tau_{n+2}^{m+1}=0,
$$

there is a solution of the difference-difference Lotka-Volterra equation (3.2),

$$
c_{n}^{m}=\frac{\boldsymbol{\tau}_{n-1}^{m} \boldsymbol{\tau}_{n+2}^{m+1}}{\boldsymbol{\tau}_{n}^{m} \boldsymbol{\tau}_{n+1}^{m+1}} .
$$

Proof. The proof is given by direct computations [12, 25].

This lemma is used for the next proposition.

Proposition 3.3. There exist nontrivial solutions of (3.2) for $n, m \in \mathbb{Z}$.

Proof. This proposition is proved by a construction of a special solution. For example, the two-soliton solution is expressed as [11, 23],

$$
\boldsymbol{\tau}_{n}^{m}=1+e^{\eta_{1}(m, n)}+e^{\eta_{2}(m, n)}+A e^{\eta_{1}(m, n)+\eta_{2}(m, n)},
$$

where $k_{a}, \omega_{a} \eta_{a}^{0}(a=1,2)$ are real numbers satisfying,

$$
\begin{gathered}
\eta_{a}(m, n)=k_{a} n-\omega_{a} m+\eta_{a}^{0}, \\
e^{\omega_{a}}=\frac{1+\delta\left(e^{k_{a}}+1\right)}{1+\delta\left(e^{-k_{a}}+1\right)}, \\
A=\frac{\sinh ^{2}\left[\left(k_{1}-k_{2}\right) / 2\right]}{\sinh ^{2}\left[\left(k_{1}+k_{2}\right) / 2\right]} .
\end{gathered}
$$

The direct substitution of (3.5) into (3.3) shows that the left-hand side of (3.3) vanishes. Using Lemma 3.2, the proposition is proved.

We note that we have more general solutions $[8,11,25]$.

4. Ultra-discrete space. Next we introduce the ultra-discrete limit following [25], which is currently studied in soliton theory. In order to make our argument easy, we will slightly modify the definition of ultra-discrete limit mentioned in [25].

Let $\overline{A_{[}[\beta]}$ be a set of nonnegative real-valued functions over $\left\{\beta \in \mathbb{R}_{>0}\right\}$ where $\mathbb{R}_{>0}$ is a set of positive real numbers.

DEFinition 4.1. We define a correspondence $\operatorname{ord}_{\beta}: \overline{\mathbb{A}_{[\beta]}} \cup\{0\} \rightarrow \mathbb{R}+\infty$. We set $\operatorname{ord}_{\beta}(0)=\infty$ for zero and for $u \in \overline{\mathbb{A}_{[\beta]}}$, if exists

$$
\operatorname{ord}_{\beta}(u):=-\lim _{\beta \rightarrow+\infty} \frac{1}{\beta} \log (u)
$$

We call this value ultra-discrete limit of $u$.

Choose a subset $\mathscr{A}_{[\beta]}$ of $\overline{\mathscr{A}_{[\beta]}}$ so that for $u \in \mathscr{A}_{[\beta]}$, $\operatorname{ord}_{\beta}(u)$ has a finite value. We identify the elements $u$ of $\overline{A_{[\beta]}}$ such that $\operatorname{ord}_{\beta}(u)=\infty$ with the zero element, $u \equiv 0$. 
Typically the ultra-discrete behaves like,

$$
\operatorname{ord}_{\beta}\left(e^{-\beta A}+e^{-\beta B}\right)=\min (A, B),
$$

for positive numbers $A$ and $B$. Hence we note that this map ord $\beta$ has the properties $\left(\mathrm{I}_{\beta}\right)$.

Proposition $4.2\left(\mathrm{I}_{\beta}\right)$. For $u, v \in \mathscr{A}_{[\beta]} \cup\{u \equiv 0\}$,

(1) $\operatorname{ord}_{\beta}(u v)=\operatorname{ord}_{\beta}(u)+\operatorname{ord}_{\beta}(v)$.

(2) $\operatorname{ord}_{\beta}(u+v)=\min \left(\operatorname{ord}_{\beta}(u), \operatorname{ord}_{\beta}(v)\right)$.

Proof. They are directly derived from Definition 4.1 (see [25]).

We note that this is a non-archimedean valuation because for $A>B$, there does not exist a finite integer $n$ such that $\operatorname{ord}_{\beta}\left(e^{-\beta A}\right)<\operatorname{ord}_{\beta}\left(n e^{-\beta B}\right)[4,14,26]$. It should be noted that this valuation is similar to the property $\mathrm{I}_{p}$ of $p$-adic valuation in Proposition 2.2. Thus we will refer to it as the ultra-valuation.

By introducing new variables $f_{n}^{m}:=-\operatorname{ord}_{\beta}\left(c_{n}^{m}\right)$ and $d:=-\operatorname{ord}_{\beta}(\delta)$ [23], we have an ultra-valuation version of the difference-difference Lotka-Volterra equation (3.2) for $c_{n}^{m} \in \mathscr{A}_{[\beta]}$ and $\delta \in \mathscr{A}_{[\beta]}$.

DEFINITION 4.3 (see [25]). The ultra-discrete difference-difference Lotka-Volterra equation is given by

$$
f_{n}^{m+1}-f_{n}^{m}=\max \left(0, f_{n-1}^{m}+d\right)-\max \left(0, f_{n+1}^{m+1}+d\right)
$$

for $\left\{f_{n}^{m} \in \mathbb{R} \mid n, m \in \Omega\right\}$ and $\delta \in \mathbb{R}$.

This equation also has the trivial solution in which all $f_{n}^{m}$ 's vanish.

Proposition 4.4. The ultra-discrete difference-difference Lotka-Volterra equation has a nontrivial solution.

Proof. We scale these variables $k_{a}, \omega_{a}$, and $\eta_{a}^{0}$ in (3.6) by $\beta$, (i.e., $\beta k_{a}, \beta \omega$, and $\left.\beta \eta_{a}^{0}\right)$ and define $\delta:=e^{-\beta}$ [23]. Then $f_{n}^{m}:=-\operatorname{ord}_{\beta}\left(c_{n}^{m}\right)$ and $d:=-\operatorname{ord}_{\beta}(\delta)$ for (3.4) and (3.5) satisfy

$$
f_{n}^{m}-f_{n}^{m+1}=\operatorname{ord}_{\beta}\left(1+\delta c_{n-1}^{m}\right)-\operatorname{ord}_{\beta}\left(1+\delta c_{n+1}^{m+1}\right) .
$$

Equation (4.3) is reduced from (4.4). Propositions 3.3 and 4.2 show this proposition.

This is also an integrable equation [25]. Of course, when the $f$ 's are given by quantities of integers times $d$, respectively, we can normalize it as $d=1$ by dividing by $d$. Then the range of these solutions is given by the set of integers $\mathbb{Z}$. However, we also remark that the set $\left\{f_{n}^{m}\right\}$ does not have the ring structure induced from (4.3); it has only a structure of additive groups. Thus the set is not directly concerned with integer theory as a theory of commutative rings.

5. $p$-adic difference-difference Lotka-Volterra equation. In this section, we show that even in the $p$-adic space, difference-difference Lotka-Volterra equation can be defined.

First we formally introduce a $p$-adic difference-difference Lotka-Volterra equation for a $p$-adic series $\left\{c_{n}^{m} \in \mathbb{Q}_{p}\right\}(p \neq 2)$. 
DEFINITION 5.1. We define the $p$-adic difference-difference Lotka-Volterra equation for a $p$-adic series $\left\{c_{n}^{m} \in \mathbb{Q}_{p}\right\}(p \neq 2)$,

$$
\frac{c_{n}^{m+1}}{c_{n}^{m}}=\frac{1+\delta_{p} c_{n-1}^{m}}{1+\delta_{p} c_{n+1}^{m+1}}
$$

where $\delta_{p} \in p \mathbb{Z}_{p}$.

Proposition 5.2. There exist nontrivial solutions of (5.1) for $n, m \in \mathbb{Z}$, which differs from a solution of all constant values $c_{n}^{m}=c$ for all $n$ and $m$.

Proof. This proposition is also proved by a construction of a special solution. Since the formal function structure (5.1) and (3.2) are the same. Thus it is obvious that (5.1) is also formally reduced to a bilinear equation; the set $\left\{c_{n}^{m}\right\}$, whose element given by

$$
c_{n}^{m}=\frac{\boldsymbol{\tau}_{n-1}^{m} \boldsymbol{\tau}_{n+2}^{m+1}}{\boldsymbol{\tau}_{n}^{m} \boldsymbol{\tau}_{n+1}^{m+1}},
$$

is a formal solution of (5.1) if $\tau_{n}^{m}$ 's satisfy

$$
\tau_{n+1}^{m+1} \tau_{n}^{m}-(1+\delta) \tau_{n+1}^{m} \tau_{n}^{m+1}+\delta \tau_{n-1}^{m} \tau_{n+2}^{m+1}=0 .
$$

Further there formally exists the two-soliton solution

$$
\boldsymbol{\tau}_{n}^{m}=1+e^{\eta_{1}(m, n)}+e^{\eta_{2}(m, n)}+A e^{\eta_{1}(m, n)+\eta_{2}(m, n)},
$$

where

$$
\begin{gathered}
\eta_{a}(m, n)=k_{a} n-\omega_{a} m+\eta_{a}^{0}, \\
e^{\omega_{a}}=\frac{1+\delta_{p}\left(e^{k_{a}}+1\right)}{1+\delta_{p}\left(e^{-k_{a}}+1\right)}, \\
A=\frac{\sinh ^{2}\left[\left(k_{1}-k_{2}\right) / 2\right]}{\sinh ^{2}\left[\left(k_{1}+k_{2}\right) / 2\right]} .
\end{gathered}
$$

Accordingly, we must check the well-definedness of these formal solutions.

Noting that from Remark 2.4(3), $p \mathbb{Z}_{p}$ is the domain of the exponential function and $1+p \mathbb{Z}_{p}$ is the domain of the logarithm function [26]. Further addition of elements of $p \mathbb{Z}_{p}$ belongs to $p \mathbb{Z}_{p}$ because $p \mathbb{Z}_{p}$ is an ideal $[16,26]$.

Let $k_{a}$ and $\eta_{a}^{0}(a=1,2)$ be elements of $p \mathbb{Z}_{p}$. Suppose that $\omega_{a}(a=1,2)$ belongs to $p \mathbb{Z}_{p}$. Then $\eta_{a}(m, n)$ in (5.5) belongs to $p \mathbb{Z}_{p}$ by the properties of ideal, and is in a domain of exponential function in $p$-adic space. Hence $\exp \left(\eta_{a}(m, n)\right)$ and $\exp \left(\eta_{1}(m, n)+\right.$ $\left.\eta_{2}(m, n)\right)$ have values as functions over $p$-adic field.

Thus consider the value of $\omega_{a}$. On the transcendental equation of $\omega_{a}$ for a given $k_{a}$ in (5.5),

$$
\omega_{a}=\log \frac{1+\delta_{p}\left(e^{k_{a}}+1\right)}{1+\delta_{p}\left(e^{-k_{a}}+1\right)},
$$

$\left(1+\delta_{p}\left(e^{k a}+1\right)\right) /\left(1+\delta_{p}\left(e^{-k a}+1\right)\right)$ can be expanded in $p$-adic space and belongs to $1+p \mathbb{Z}_{p}$. Since the range of the logarithm function for $1+p \mathbb{Z}_{p}$ is $p \mathbb{Z}_{p}, \omega_{a}$ has a value in $p \mathbb{Z}_{p}$. Hence the above assumption that $\omega_{a}$ belongs to $p \mathbb{Z}_{p}$ is correct.

Similarly, using $p \neq 2,\left(k_{1} \pm k_{2}\right) / 2$ belongs to $p \mathbb{Z}_{p}$ and sine-hyperbolic function of $p \mathbb{Z}_{p}$ has a value in $1+p \mathbb{Z}_{p}$. Thus $A$ in (5.5) can also be computed. 
Hence the $p$-adic version $\tau_{n}^{m}$ in (5.4) and $c_{n}^{m}$ has a finite value in $p$-adic space. In other words, one- and two-soliton solutions exist in (5.1).

Furthermore, we can construct other soliton solutions for $p$-adic equation (5.1) following the procedure in $[6,7,8,11,25]$.

As the $p$-adic difference-difference Lotka-Volterra equation is well defined, we can consider the $p$-adic valuation of the equation.

Proposition 5.3. For the solutions of the $p$-adic difference-difference Lotka-Volterra equation $c_{n}^{m}$ (5.1), we let $f_{n}^{m}:=-\operatorname{ord}_{p}\left(c_{n}^{m}\right)$ and $d_{p}:=-\operatorname{ord}_{p}\left(\delta_{p}\right)$.

(1) (5.1) is reduced to

$$
f_{n}^{m}-f_{n}^{m+1}=\operatorname{ord}_{p}\left(1+\delta_{p} c_{n-1}^{m}\right)-\operatorname{ord}_{p}\left(1+\delta_{p} c_{n+1}^{m+1}\right) .
$$

(2) When $f_{n}^{m} \neq-d_{p}$, (5.7) becomes

$$
f_{n}^{m+1}-f_{n}^{m}=\max \left(0, f_{n-1}^{m}+d_{p}\right)-\max \left(0, f_{n+1}^{m+1}+d_{p}\right) .
$$

Proof. The proof is obvious.

REMARK 5.4. (1) It is also surprising that (5.8) has the same form as the ultradiscrete difference-difference Lotka-Volterra equation (4.3). We conclude that the structure of the ultra-discrete limit has the same as that in $p$-adic analysis.

(2) The well-definedness of the $p$-adic Lotka-Volterra equation is also valid for the case of $p=2$. For the case of $p=2$, since $4 \mathbb{Z}_{2}$ is the domain of exponential function, $\delta_{2}, k_{a}$, and $\eta_{a}^{0}(a=1,2)$ belong to $4 \mathbb{Z}_{2}$. Further, though $k_{a}$ must also be satisfied with $k_{1} \pm k_{2} \in 8 \mathbb{Z}_{2}$, we can argue it in a similar way.

6. Ultra-discrete metric from the point of view of valuation theory. As we saw the similarity between ultra valuation and $p$-adic valuation, we will construct the ultrametric following the definition of $p$-adic metric.

Since soliton theory is defined over the field whose characteristic is zero, we might regard it as a theory over $\mathbb{Q}_{\infty}$. However, it should be also noted that since the ultravaluation is a natural non-archimedean valuation of $\mathscr{A}_{[\beta]}$, another real-valued metric is naturally defined in $\mathscr{A}_{[\beta]}$, which differs from the ordinary metric $|x|_{\infty} \equiv|x|$ in $\mathbb{Q}_{\infty}$.

By introducing a real number $\bar{\beta} \gg 1$, a quantity is defined for $x \in \mathscr{A}_{[\beta]} \cup\{u \equiv 0\}$ as,

$$
|x|_{\beta}:=\left(e^{-\bar{\beta}}\right)^{\operatorname{ord}_{\beta}(x)},
$$

which is a kind of exponential valuation [14]. We call this ultrametric since it is a metric.

Proposition 6.1 $\left(\mathrm{II}_{\beta}\right)$. For $u, v \in \mathscr{A}_{[\beta]} \cup\{u \equiv 0\}$, the ultrametric $|u|_{\beta}$ and $|v|_{\beta}$ have the following properties:

(1) $|u|_{\beta}$ depends upon $\bar{\beta}$.

(2) If $|v|_{\beta}=0, v=0$.

(3) $|v|_{\beta} \geq 0$.

(4) $|v u|_{\beta}=|v|_{\beta}|u|_{\beta}$.

(5) $|u+v|_{\beta} \leq|u|_{\beta}+|v|_{\beta}$. 
Proof. They are obvious from definition (6.1).

REMARK 6.2. (1) The ultra-discrete limit and the $p$-adic valuation are given by

$$
\operatorname{ord}_{\beta}(u)=\lim _{\beta \rightarrow+\infty} \log _{e^{-\beta}}(u), \quad \operatorname{ord}_{p}(v)=\log _{p}[[v]]_{p},
$$

for $u \in \mathscr{A}_{[\beta]}$ and $v \in \mathbb{Q}_{p},(u \neq 0, v \neq 0)$. Here $\left.e^{-\beta}\right|_{\beta \rightarrow \infty}$ plays the same role of $p$. However it should be noted that since this ultra valuation is defined in $\mathbb{R},|x|_{\beta}$ is defined by $\left(e^{-\bar{\beta}}\right)^{\operatorname{ord}_{\beta}(x)}$ rather than $\left(e^{-\bar{\beta}}\right)^{-\operatorname{ord}_{\beta}(x)}$ whereas $|x|_{p}=p^{-\operatorname{ord}_{p} x}$.

(2) Since $x \in \mathscr{A}_{[\beta]}$ has a finite value at $\beta \rightarrow \infty$, we have the relation

$$
\left.\left.|x|_{\beta}\right|_{\bar{\beta} \sim \infty} \sim \exp \left(-\bar{\beta}\left(-\frac{\log x}{\beta}\right)\right)\right|_{\bar{\beta} \sim \beta \sim \infty}=\left.|x|^{\bar{\beta} / \beta}\right|_{\bar{\beta} \sim \beta \sim \infty} .
$$

As it is not completely guaranteed, it may be regarded that $|x|_{\beta} \sim|x|$, in essence, by synchronizing $\bar{\beta}$ and $\beta$ in (6.3). It implies that the ultrametric $|x|_{\beta}$ might be equivalent to the natural metric at $\mathbb{Q}_{\infty}$.

(3) In this metric, the convergence condition of series is also equivalent with the vanishing condition of sequences and we have the relation,

$$
\left|\sum_{m} x_{m}\right|_{\beta}=e^{-\bar{\beta} \min \left(\operatorname{ord}_{\beta}\left(x_{m}\right)\right)} .
$$

We should note that this metric appears in the low temperature treatment of statistical physics and in the semi-classical treatment of path integral [9, 10]. For the low temperature limit $\bar{\beta} \sim \beta=1 / T, T \rightarrow 0$ or the classical limit of deformation parameter $\bar{\beta} \sim \beta=1 / \hbar, \hbar \rightarrow 0$, only the minimal point survives and contributes to zero temperature or classical phenomena.

Thus although the ultra-discrete limit is sometimes called "quantization," as a terminology of discretization in digital picture, in the soliton theory, it should be regarded as low temperature limit of statistical mechanical phenomena or classical limit of quantum phenomena. (The reason why the domain of $\mathscr{A}_{[\beta]}$ must be nonnegative might be related to the positiveness of the probability.)

From quantum mechanical point of view, it must be emphasized that the classical regime appears as a non-archimedean valuation, which is an algebraic manipulation. It implies that classical limit might be regarded as valuation of a localized ring.

(In this analogy, we might regard that $\mathbb{Z}$ is in a classical regime whereas $\mathbb{Q}_{p}$ 's $(p \in \mathfrak{A})$ are of quantum world in number theory.)

(4) It is known that some of the properties in the $q$ analysis can be regarded as those in $p$-adic analysis by setting $q=1 / p$ [26]. We have correspondences among $p, q$, and $e^{\beta}$ as,

$$
e^{-\beta} \Longleftrightarrow p(\beta \sim \infty), \quad p \Longleftrightarrow \frac{1}{q}, \quad q \Longleftrightarrow e^{\beta}(\beta \sim 0) .
$$

(5) There might arise a question why the ultra-discrete limit is related to integervalued solutions for a soliton equation. Function form of finite type solution of (3.1) including soliton solution is completely determined at the infinity point of the spectral parameters $k=\infty[6,15]$. The soliton solution is given by exponential functions 
whose power is polynomial of $(k, s, t)$ owing to the algebraic properties of soliton solutions. Since polynomial of integer-valued $(k, s, t)$ is also integer, ultra-discrete limit is associated with integer-valued solutions.

Finally, we hope that the correspondence between $p$-adic and ultra-discrete structures might have an effect on the studies of relations between soliton theory and number theory $[13,19]$ and more generally between physics and number theory [2, 3, $5,20,26]$.

Acknowledgements. I would like to thank Prof T. Tokihiro, Prof Y. Ônishi, and members of Toda seminar. I am also grateful to Prof K. Tamano and H. Mitsuhashi for fruitful discussions.

\section{REFERENCES}

[1] N. L. Balazs and A. Voros, Chaos on the pseudosphere, Phys. Rep. 143 (1986), no. 3, 109240. MR 88h:58070.

[2] J.-B. Bost and A. Connes, Hecke algebras, type III factors and phase transitions with spontaneous symmetry breaking in number theory, Selecta Math. (N.S.) 1 (1995), no. 3, 411-457. MR 96m:46112. Zbl 842.46040.

[3] L. Brekke and P. G. O. Freund, p-adic numbers in physics, Phys. Rep. 233 (1993), no. 1, 1-66. MR 94h:11115.

[4] J. W. S. Cassels, Lectures on Elliptic Curves, London Mathematical Society Student Texts, vol. 24, Cambridge University Press, Cambridge, 1991. MR 92k:11058. Zbl 752.14033 .

[5] A. Connes, Formule de trace en géométrie non-commutative et hypothèse de Riemann [Trace formula in noncommutative geometry and the Riemann hypothesis], C. R. Acad. Sci. Paris Sér. I Math. 323 (1996), no. 12, 1231-1236 (French). MR 97k:11124. Zbl 864.46042.

[6] E. Date, M. Jimbo, and T. Miwa, Method for generating discrete soliton equations. I, J. Phys. Soc. Japan 51 (1982), no. 12, 4116-4124. MR 84k:58103a.

[7] _ Method for generating discrete soliton equations. II, J. Phys. Soc. Japan 51 (1982), no. 12, 4125-4131. MR 84k:58103a.

[8]___ Method for generating discrete soliton equations. III, J. Phys. Soc. Japan 52 (1983), no. 2, 388-393. MR 84k:58103b. Zbl 571.35105.

[9] P. A. M. Dirac, The Principles of Quantum Mechanics, 4th ed., International Series of Monographs on Physics, Oxford University Press, Oxford, 1958. Zbl 0080.22005.

[10] R. P. Feynman and A. R. Hibbs, Quantum Mechanics and Path Integrals, International Series in Pure and Applied Physics, McGraw-Hill, Auckland, 1965. Zbl 176.54902.

[11] R. Hirota, Nonlinear partial difference equations. I. A difference analogue of the Kortewegde Vries equation, J. Phys. Soc. Japan 43 (1977), no. 4, 1424-1433. MR 57\#925a.

[12] R. Hirota and S. Tsujimoto, Conserved quantities of a class of nonlinear differencedifference equations, J. Phys. Soc. Japan 64 (1995), no. 9, 3125-3127. MR 96i:39020.

[13] S. Ishiwata, S. Matsutani, and Y. Ônishi, Localization state of hard core chain and cyclotomic polynomial: hard core limit of diatomic Toda lattice, Phys. Lett. A 231 (1997), no. 3-4, 208-216. MR 98g:82010.

[14] K. Iwasawa, Algebraic Function Theory, Iwanami, Tokyo, 1952 (Japanese).

[15] I. M. Krichever, Methods of algebraic geometry in the theory of non-linear equations, Russian Math. Surveys 32 (1977), no. 6, 185-213. Zbl 0386.35002.

[16] K. Lamotke (ed.), Zahlen, Grundwissen Mathematik, vol. 1, Springer-Verlag, Berlin, 1983 (German). MR 86b:00001. Zbl 543.00001.

[17] J. J. Morales and C. Simó, Picard-Vessiot theory and Ziglin's theorem, J. Differential Equations 107 (1994), no. 1, 140-162. MR 95e:12009. Zbl 799.58035. 
[18] M. Mulase, Cohomological structure in soliton equations and Jacobian varieties, J. Differential Geom. 19 (1984), no. 2, 403-430. MR 86f:14016. Zbl 559.35076.

[19] M. Pigli, Adelic integrable systems, J. Math. Phys. 36 (1995), no. 12, 6829-6845. MR 97b:11072. Zbl 858.39003.

[20] R. Rammal, G. Toulouse, and M. A. Virasoro, Ultrametricity for physicists, Rev. Modern Phys. 58 (1986), no. 3, 765-788. MR 87k:82105.

[21] K. Sogo, Time-dependent orthogonal polynomials and theory of soliton. Applications to matrix model, vertex model and level statistics, J. Phys. Soc. Japan 62 (1993), no. 6, 1887-1894. MR 95a:81255.

[22] K. Sunada, Laplacian to Kihongun (Laplacian and Fundamental Group), Kinokuniya, Tokyo, 1985 (Japanese).

[23] D. Takahashi, Ultra-discrete Toda lattice equation-A grandchild of Toda, Advances in Soliton Theory and its Applications (The 30th Anniversary of the Toda Lattice) (Graduate University for Advanced Studies, Hayama Campus), 1996, pp. 36-37.

[24] D. Takahashi and J. Satsuma, A soliton cellular automaton, J. Phys. Soc. Japan 59 (1990), no. 10, 3514-3519. MR 91i:58075.

[25] T. Tohikiro, D. Takahashi, J. Matsukidaira, and J. Satsuma, From soliton equations to integrable cellular automata through a limiting procedure, Phys. Rev. Lett. 76 (1996), no. $18,3247-3250$.

[26] V. S. Vladimirov, I. V. Volovich, and E. I. Zelenov, p-adic Analysis and Mathematical Physics, Series on Soviet and East European Mathematics, vol. 1, World Scientific Publishing, New Jersey, 1994. MR 95k:11155.

SHigeKi MATSUTANI: 8-21-1 HigaShi-LinKAN SAgAmiHARA, 228-0811, JAPAN

E-mail address: rxbo1142@nifty.ne.jp 


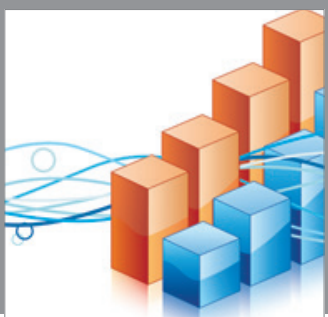

Advances in

Operations Research

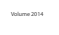

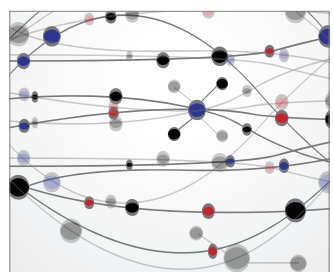

\section{The Scientific} World Journal
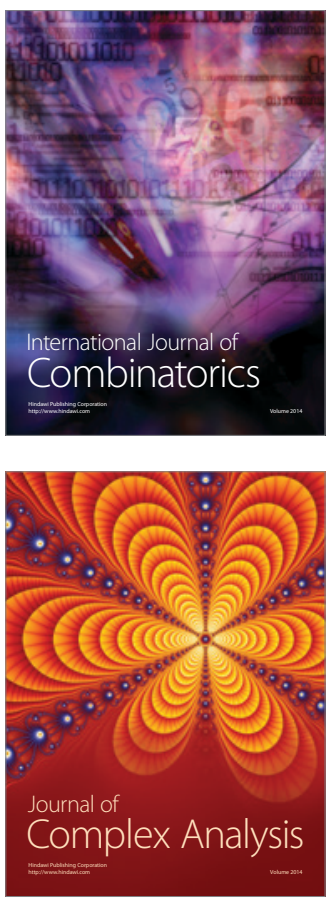

International Journal of

Mathematics and

Mathematical

Sciences
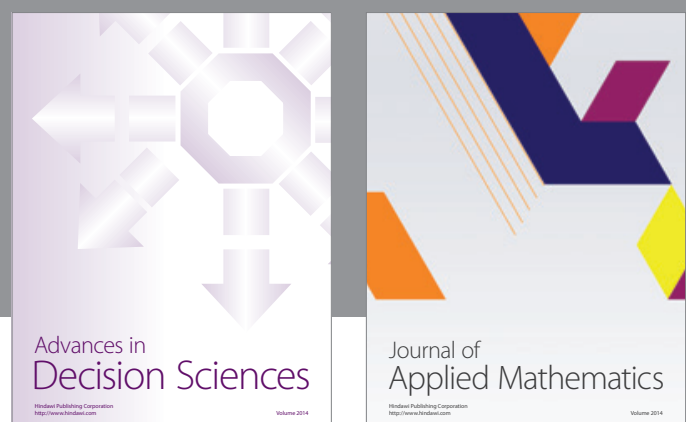

Journal of

Applied Mathematics
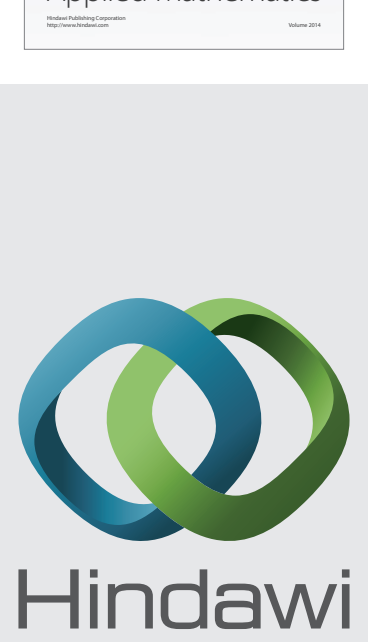

Submit your manuscripts at http://www.hindawi.com
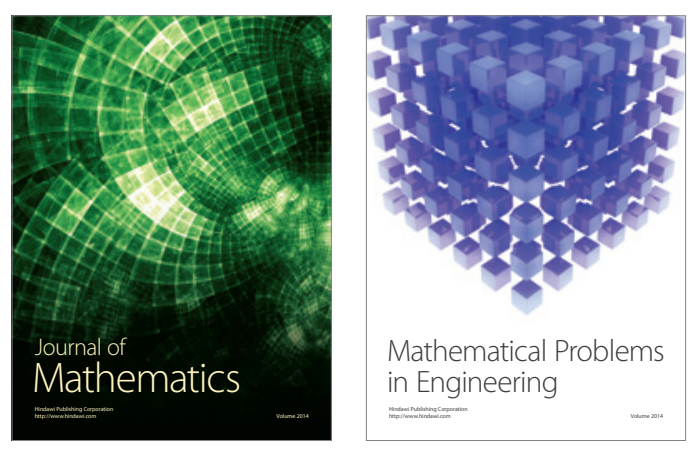

Mathematical Problems in Engineering
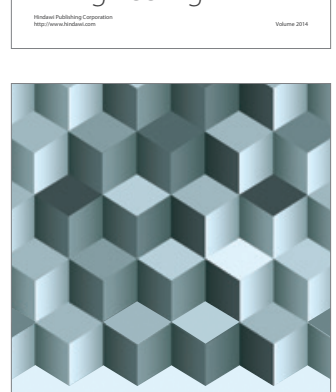

Journal of

Function Spaces
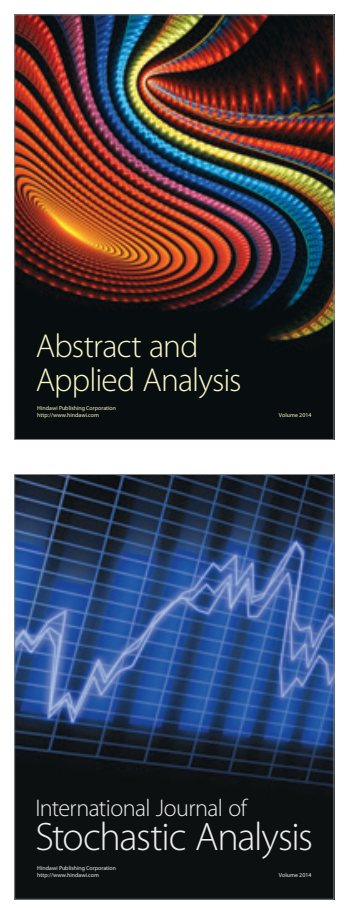

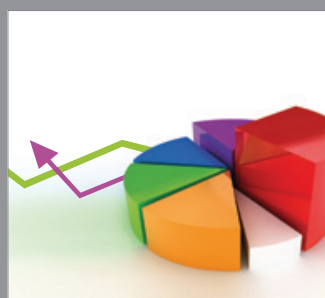

ournal of

Probability and Statistics

Promensencen
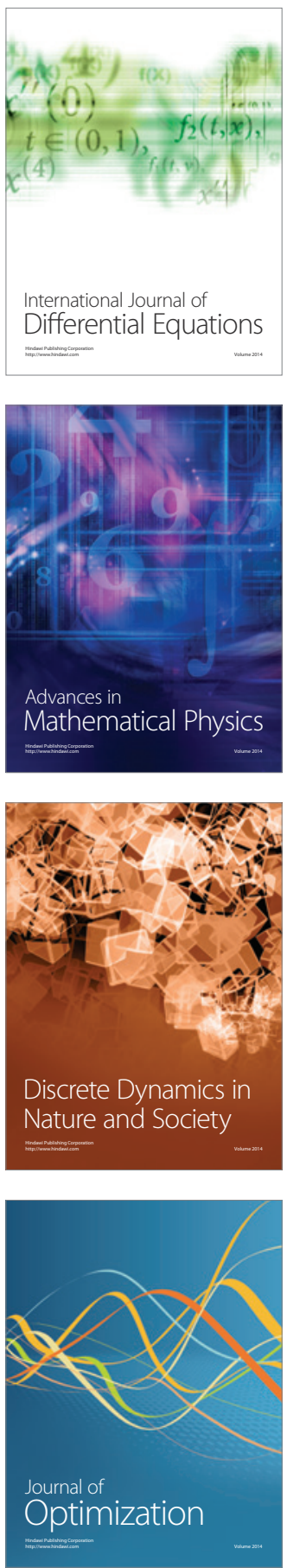